\title{
Plantas no nativas naturalizadas de la Península de Baja California, MÉXico
}

\author{
Pedro P. Garcillán ${ }^{1}{ }^{4}$, José Luis León De La Luz¹, Jon P. Rebman² y José Delgadillo 3 \\ ${ }^{1}$ Centro de Investigaciones Biológicas del Noroeste, La Paz, Baja California, México \\ ${ }^{2}$ San Diego Natural History Museum, San Diego, California, EEUU \\ ${ }^{3}$ Facultad de Ciencias, Universidad Autónoma de Baja California, Ensenada, Baja California, México \\ ${ }^{4}$ Autor para la correspondencia: ppgarcillan@cibnor.mx
}

\begin{abstract}
Resumen: Se presenta un inventario exhaustivo de la flora no nativa naturalizada de la península de Baja California. Se parte de la relación de especies referidas a la península de Baja California por Espinosa-García et al. (2000) en su base de datos Malezas introducidas en México, además se revisó extensamente la literatura científica publicada acerca de la flora o vegetación de alguna parte o del conjunto de la península. Se eliminó a las especies sin respaldo en especímenes de herbario. No se consideraron especies casuales, ni presentes exclusivamente como cultivos. Se analizó su origen geográfico, formas de vida, ciclo de vida y estatus invasor. Los resultados muestran 233 taxones no nativos naturalizados en la península de Baja California, pertenecientes a 230 especies, 149 géneros y 37 familias. La familia Poaceae contiene casi un tercio (31\%) de las especies, seguida por Asteraceae (13\%), Brassicaceae ( $8 \%$ ) y Chenopodiaceae (5\%). La mayoría de las especies proceden de la Cuenca del Mediterráneo y Eurasia (38\%). La forma de vida herbácea es dominante (91\%), frente a sólo $6 \%$ de arbóreas y $3 \%$ de arbustivas. La mayoría de las especies son de ciclo de vida anual (54\%), frente a un 34\% de especies perennes. Se identificaron a 18 especies (8\% del total) con comportamiento invasor en la península de Baja California.
\end{abstract}

Palabras clave: Baja California, flora, plantas invasoras, plantas no nativas.

\begin{abstract}
We present a comprehensive inventory of naturalized non-native flora of the Baja California peninsula. As a starting point, we used the list of species referred to Baja California peninsula by Espinosa-García et al. (2000) in his database Malezas Introducidas en México, and then exhaustively reviewed the scientific bibliography for floristic flora and vegetation publications on various parts and the entire peninsula. We eliminated those reported plant taxa that lack specimens deposited in herbaria. Casual species (waifs) or species only found as cultivated plants were not included. We analyzed their geographic origin, life form, life cycle, and invasive status. We found 233 naturalized non-native taxa on the Baja California peninsula belonging to 230 species, 149 genera and 37 families. Poaceae has almost one-third of the total species (31\%), followed by Asteraceae (13\%), Brassicaceae $(8 \%)$, and Chenopodiaceae (5\%). The majority of the non-native plants are of Mediterranean and Euroasiatic origin (42\%). Herb is the dominant life form (91\%) and only 6\% are trees and 3\% are shrubs. Most species are annuals (54\%), while 34\% are perennial species. We identified 18 species (8\% of the total) as highly invasive species in the Baja California peninsular region.
\end{abstract}

Key words: Baja California, flora, invasive plants, non-native plants.

$\mathbf{L}$ a expansión de las actividades humanas, a nivel del planeta, se ha intensificado dramáticamente en los últimos siglos (Turner II et al., 1990; Vitousek et al., 1997). El movimiento de especies asociado directa o indirectamente a dichas actividades (especies exóticas, introducidas o no nativas sensu Pyšek et al., 2004), ha aumentado incesantemente en los últimos siglos, tanto en su número como en su alcance geográfico (Mack et al., 2000; Mack y Lonsdale, 2001). Este intercambio biológico afecta a la diversidad y el funcionamiento de los sistemas ecológicos a distintos niveles (Sax y
Gaines, 2003; Ehrenfeld, 2010), así como en las actividades económicas (Pimentel et al., 2000, 2005; Vilà et al., 2009); pero también ofrece estimulantes oportunidades de estudio de procesos ecológicos, biogeográficos o evolutivos (Sax et al., 2005; McMahon et al., 2006; Richardson, 2011). Desde la publicación en torno a 1960 de las dos obras fundacionales del área de las invasiones biológicas: Elton (1958), y Baker y Stebbins (1965); el interés sobre el tema y la conciencia de sus implicaciones no ha dejado de crecer (Mooney y Hobbs, 2000; Mack et al., 2000; Sax et al., 2005; Richardson, 2011). 
Los inventarios regionales de especies no nativas constituyen una información clave en la comprensión de las invasiones biológicas. La disponibilidad de listas de especies no nativas para una región determinada permite la realización de estudios comparativos y ayuda a entender los patrones taxonómicos y biogeográficos de la invasión de plantas (Pyšek, 1998; Sax, 2001; Villaseñor y Espinosa-García, 2004; Pyšek y Richardson, 2006). Asimismo, constituyen el punto de partida para explorar los patrones históricos de la invasión (Pyšek et al., 2003; Sol et al., 2008), analizar la existencia de atributos de las especies no nativas que puedan determinar su capacidad de colonización (Sutherland, 2004; Pyšek y Richardson, 2007) y la susceptibilidad de distintos ecosistemas a ser invadidos (Davis et al., 2000, 2005; Richardson y Pyšek, 2006). La disponibilidad de esta información base es de gran ayuda para desarrollar estrategias de manejo y control (Hulme et al., 2009; Khuroo et al., 2008).

La información acerca de especies no nativas en la península de Baja California se puede remontar a las primeras crónicas de la época misional (del Barco, 1780). Sin embargo, su presencia en los estudios florísticos ha sido marginal. En el caso de las plantas, la única información sobre especies no nativas a nivel peninsular está contenida en la flora publicada por Ira Wiggins hace ya más de tres décadas (Wiggins, 1980), donde señala explícitamente 168 taxones introducidos o escapados de cultivos (6\% del total de 2,705 taxones contenidos en dicha flora); y en la monografía sobre la familia Poaceae para la región (74 taxones) publicada por Gould y Moran (1981). León de la Luz et al. (2009) publicaron una primera aproximación de las malezas (i. e., especies colonizadoras de zonas perturbadas), tanto nativas (85 taxones) como no nativas (58 taxones), para el estado de Baja California Sur. El resto de la información existente sobre plantas introducidas o invasoras está dispersa en trabajos de diversa naturaleza y alcance geográfico. Compilaciones de plantas no nativas existen ya para el conjunto de México (Villaseñor y Espinosa-García, 2004) y para regiones circundantes a la península de Baja California como Sonora (Van Devender et al., 2009) y California (Bossard et al., 2000; Hrusa et al., 2002; Cal-IPC, 2006, 2007; Dean et al., 2008). Sin embargo, no existe aún una lista de especies no nativas para la península de Baja California.

El objetivo del presente estudio fue elaborar una lista de las especies no nativas naturalizadas de la península de Baja California, explorar sus afiliaciones biogeográficas e indicar aquellas especies en que se ha observado comportamiento invasor. Esta información básica no pretende ser definitiva, sino servir como un punto de partida que ha de ser necesariamente revisado y actualizado en futuras evaluaciones.

\section{Materiales y métodos}

Área de estudio. En este estudio se consideró la península de Baja California como el área delimitada por los dos estados que la conforman (Baja California y Baja California Sur), así como sus islas correspondientes del Golfo de California y del Pacífico, incluida Isla Guadalupe. Esta región se extiende a lo largo de casi 9 grados de latitud, desde $22^{\circ}$ $52^{\prime}$ a $32^{\circ} 42^{\prime} \mathrm{N}$, con una extensión de $145,359 \mathrm{~km}^{2}$, y una línea de costa de 3,624 km (33\% de la costa total de México) (INEGI, 2011). Este largo apéndice de tierra, dispuesto en paralelo al continente, contiene en 1,300 km de longitud una singular réplica en miniatura de la transición de biomas que ocurre en México (Figura 1): desde la región tropical con lluvias de verano en su extremo sur, hasta la vegetación de carácter mediterráneo con precipitación de invierno en el noroeste peninsular; conectadas ambas por una región desértica de escasa precipitación y variable proporción de su estacionalidad, que constituye a su vez, el brazo peninsular del Desierto Sonorense (Hastings y Turner, 1965; Garcillán et al., 2010). Esta diversidad de biomas, conjuntamente con la variedad topográfica y climática actual, y la compleja historia geológica y climática han generado una región de gran heterogeneidad ecológica (González-Abraham et al., 2010).

Se considera que los primeras poblaciones humanas se asentaron en la península hace aproximadamente 10,000 años (Davis, 2003; Fujita, 2006), y se estima que el tamaño

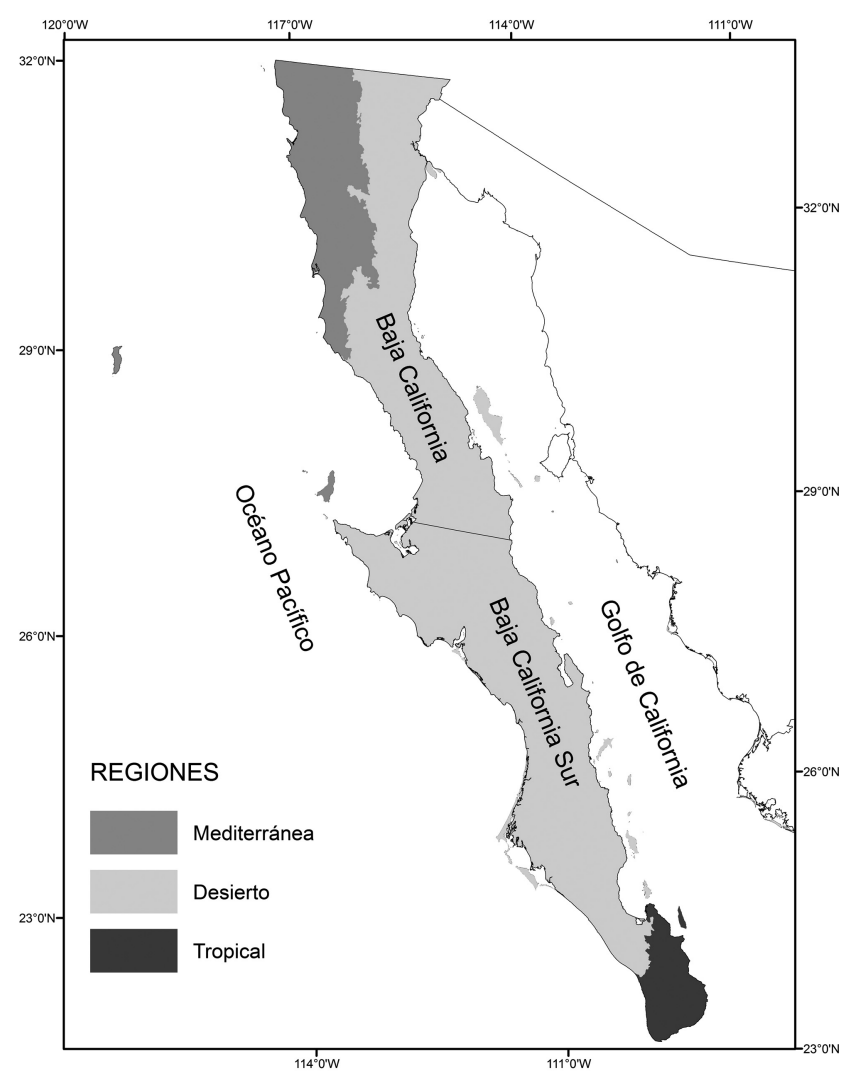

Figura 1. Península de Baja California y sus tres grandes regiones bióticas: Mediterránea, Desértica y Tropical, de acuerdo a González-Abraham et al., (2010). 
de la población peninsular fue relativamente bajo (aprox. 50,000 personas) hasta el inicio del establecimiento europeo en 1697 (Aschmann, 1959). A partir de esta fecha, la población nativa decreció drásticamente y la densidad de población humana no volvió a los valores previos al establecimiento europeo hasta casi dos siglos después (Deasy y Gerhard, 1944). Además del impacto sobre la población humana nativa, el establecimiento europeo en la península significó la introducción de la Revolución Neolítica; esto es, la agricultura y la ganadería por primera vez en la historia peninsular. En la segunda mitad del siglo pasado la región experimentó un intenso incremento poblacional; la población pasó de 130,378 hab. en 1940 a 3,792,096 hab. en 2010; es decir, se multiplicó por 29 (INEGI, 2010, 2011). Este aumento poblacional llevó aparejado una intensificación del uso del territorio (especialmente urbano y agrícola) y una expansión en el desarrollo de las comunicaciones terrestres, marítimas y aéreas de la región a nivel nacional e internacional. Si consideramos que la llegada y naturalización de nuevas especies están correlacionadas con la intensidad de las actividades humanas (Taylor e Irwin, 2004; Meyerson y Mooney, 2007; Pyšek et al., 2010), las últimas décadas han debido ser por tanto un periodo de intenso intercambio biológico en la península.

Terminología. En términos biogeográficos, un taxón (especie, subespecie) no nativo de una región es aquél cuya área nativa de distribución está fuera de las fronteras definidas para dicha región (península de Baja California en este estudio), y cuya presencia en la misma se considera relacionada directa o indirectamente a las actividades humanas (= exótico, introducido; Richardson et al., 2000; Pyšek et al., 2004). Si un taxón es nativo para alguna parte de la península, no es considerado como exótico, independientemente de si ha sido introducido localmente en otras áreas peninsulares. Este grupo de taxones nativos "translocados" regionalmente son de enorme interés ecológico y merecen sin duda un estudio propio. Siguiendo la terminología propuesta por Richardson et al. (2000) y Pyšek et al. (2004), este trabajo sólo se refiere a taxones no nativos presentes fuera de cultivo y que se consideran naturalizados; es decir, con poblaciones autosustentables sin intervención humana directa. Ni los taxones casuales fuera de cultivo, ni los cultivados han sido incluidos. La frontera entre categorías no es siempre clara, y siguiendo un criterio conservador al respecto, se han descartado los casos dudosos.

Origen geográfico. Se determinó el carácter nativo o no de cada taxón y su origen geográfico mediante el uso de diversas fuentes electrónicas disponibles, como Global Compendium of Weeds <www.hear.org/gcw/ $>$, CalFlora $<$ www. calflora.org >, Malezas de México (Vibrans, 2011) y diversas floras electrónicas internacionales (eFloras, 2011). Siguiendo la sugerencia de Pyšek et al. (2002) se describe el ori- gen de los taxones en términos continentales: Eurasia, Asia, Europa (Urales-Mar Caspio-Cáucaso como límite oriental aproximado), Cuenca Mediterránea, África (incluyendo aquellas especies de distribución limitada a África del Norte o África del Sur), Norteamérica, Sudamérica y Australia. Sin embargo, se señala aquellos taxones que aunque se distribuyen de manera natural en México y/o sur de los Estados Unidos, se consideran que son exóticos en la península de Baja California. Finalmente, se categorizó como de amplia distribución aquellos taxones cuya área nativa de distribución abarca tres o más continentes, y como criptogénicos aquellos casos en que su origen biogeográfico es oscuro.

Se calificaron como invasores a aquellos taxones que según las observaciones en campo muestran densidades altas (al menos localmente); así como una alta capacidad de dispersión y expansión sobre grandes áreas (Richardson et al., 2000; Pyšek et al., 2004). Además, dado que el carácter invasor de una especie en otras regiones se ha considerado como un buen indicio de su capacidad de invasión en una región dada (Groves et al., 2001), se señalan como invasores potenciales a aquellos taxones que, aun no siendo observados como invasores en la península, han sido reportados como tales en California (Cal-IPC, 2006, 2007), Arizona (Northam et al., 2005) o Sonora (Van Devender et $a l ., 2009)$. Finalmente, y con base en las observaciones en campo, se indica la distribución preferente de los diferentes taxones en las tres grandes regiones de la península (mediterránea, desértica y tropical) de acuerdo a la regionalización de González-Abraham et al. (2010).

Base de datos. Una lista preliminar se construyó a partir de la relación de taxones considerados no nativos, introducidos o escapados de cultivo en la flora peninsular publicada por Wiggins (1980) y los taxones presentes en la península de acuerdo a la base de datos "Malezas Introducidas en México" de Espinosa García (2000) y cuyos resultados se publicaron en Espinosa-García et al. (2004a, b) y Villaseñor y Espinosa-García (2004). Posteriormente se hizo una exhaustiva revisión de la literatura publicada sobre flora o vegetación de alguna parte o del conjunto de la península y se añadieron los taxones señalados en las mismas como introducidos, exóticos o no nativos. Se incorporaron a esta lista provisional algunos taxones no encontrados en la bibliografía revisada, pero de cuya presencia en la península se tienen datos con base en la experiencia de campo y colecta. Finalmente, de esta lista inicial de taxones no nativos que se consideraron naturalizados (incluyendo sus sinonimias), se han eliminado aquellos que no presentaron al menos un ejemplar registrado en alguno de los tres herbarios de la región (SD, San Diego Natural History Museum, San Diego, California; BCMEX, Universidad Autónoma de Baja California, Ensenada, Baja California y HCIB, Centro de Investigaciones Biológicas del Noroeste, La Paz, Baja California Sur). La relación final resultante, que constituye la lista de 
plantas no nativas naturalizadas que se presenta en este estudio, está respaldada en su totalidad por especímenes depositados en los herbarios citados.

\section{Resultados}

Composición taxonómica. El inventario incluye 233 taxones no nativos naturalizados en la península de Baja California, pertenecientes a 230 especies, 149 géneros y 37 familias (Apéndice). Sólo tres especies presentan dos subespecies distintas, por lo que el análisis numérico se expresará en términos de especies y referido al total de 230 especies. Ocho especies son primeras citas para México (Carduus pycnocphalus, Ehrharta erecta, Erodium botrys, Gastrydium phleoides, Limonium perezii, Lythrum hyssopifolia, Malephora crocea y Myoporum laetum). La distribución taxonómica está dominada por la familia Poaceae, que con un total de 71 especies contiene casi un tercio (31\%) del total de especies introducidas. Le siguen en orden de importancia las familias Asteraceae (13\%), Brassicaceae (8\%) y Chenopodiaceae (5\%). Cinco familias suman $57 \%$ de las especies (Figura 2). Se identificaron 18 especies con comportamiento invasor ( $8 \%$ del total), distribuidas en las familias Poaceae (cinco especies), Aizoaceae (tres especies), Asteraceae (dos especies); Anacardiaceae, Apiaceae, Apocynaceae, Brassicaceae, Chenopodiaceae, Euphorbiaceae, Scrophulariaceae y Tamaricaeae presentaron sólo una especie invasora por familia. La región mediterránea, en el noroeste peninsular, es la más rica en plantas no nativas, con 171 especies (74\% del total), seguida por la región desértica con 104 especies $(45 \%)$ y la región tropical del extremo sur peninsular con sólo 58 especies $(25 \%)$.

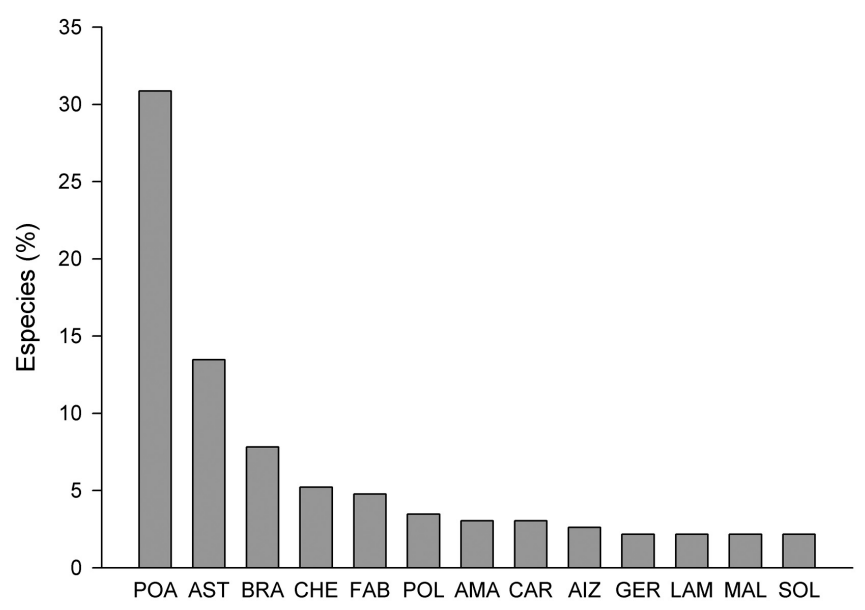

Figura 2. Distribución taxonómica de las plantas exóticas naturalizadas a nivel de familia. Sólo se muestran las familias que contienen 2\% o más del total de 230 especies. POA: Poaceae, AST: Asteraceae, BRA: Brassicaceae, CHE: Chenopodiaceae, FAB: Fabaceae, POL: Polygonaceae, AMA: Amaranthaceae, CAR: Caryophyllaceae, AIZ: Aizoaceae, GER: Geraniaceae, LAM: Lamiaceae, MAL: Malvaceae y SOL: Solanaceae.

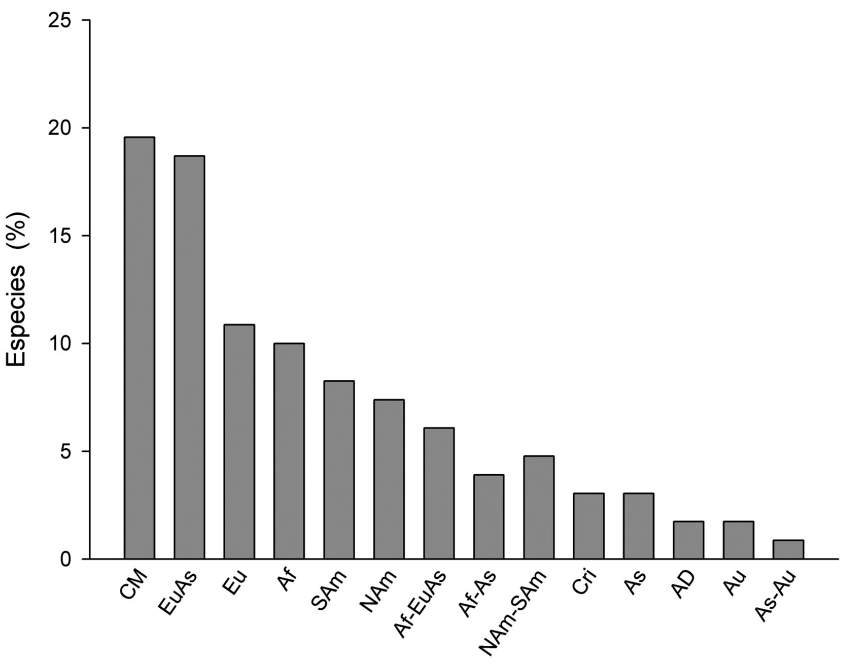

Figura 3. Distribución de las plantas exóticas naturalizadas presentes en la península de Baja California según su origen geográfico. $\mathrm{AD}=$ amplia distribución intercontinental, $\mathrm{Af}=$ África, As = Asia, $\mathrm{Au}=$ Australia, $\mathrm{CM}=$ Cuenca Mediterránea, Cri = criptogénicas, $\mathrm{Eu}=$ Europa, $\mathrm{EuAs}=$ Eurasia, $\mathrm{NAm}=$ Norteamérica y SAm $=$ Sudamérica.

Origen geográfico. Las regiones que aportan mayor número de plantas a la península de Baja California son la Cuenca del Mediterráneo (20\%) y Eurasia (19\%); seguidas por Europa (11\%), África (10\%), Sudamérica (8\%), Norteamérica (7\%) y África-Eurasia (6\%). Con aportaciones de menos del 5\% aparecieron regiones como Asia, Australia, Australasia, África-Asia, Norteamérica-Sudamérica, criptogénicas y es-

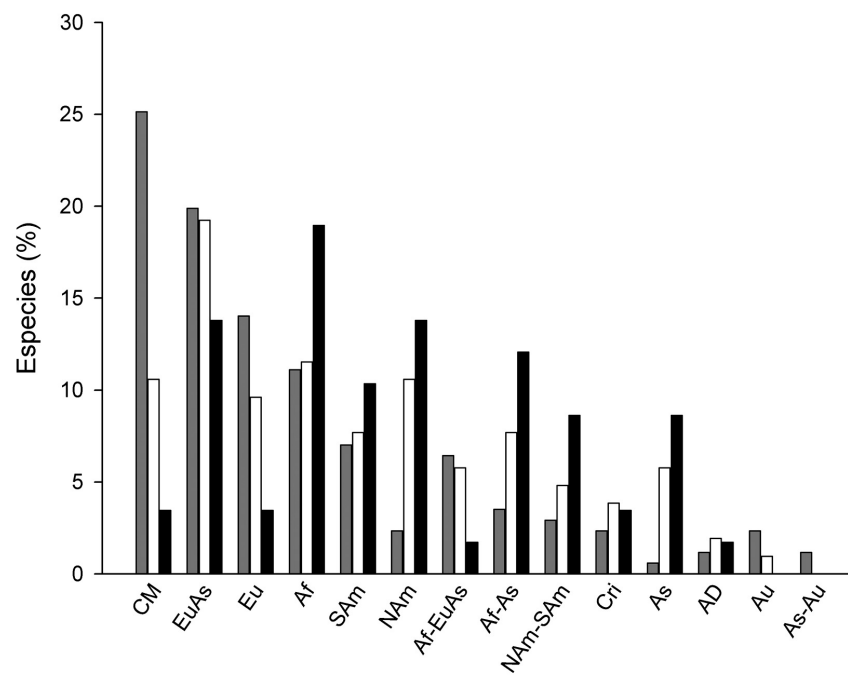

Figura 4. Distribución de las plantas exóticas naturalizadas presentes en la península de Baja California según su origen geográfico, desagregado por las tres regiones generales presentes en la península: mediterráneo (gris), desértico (blanco) y tropical (negro). AD = amplia distribución intercontinental, Af = África, As = Asia, Au = Australia, $\mathrm{CM}=$ Cuenca Mediterránea, $\mathrm{Cri}$ = criptogénicas, Eu = Europa,

EuAs $=$ Eurasia, NAm = Norteamérica y SAm = Sudamérica. 
pecies de amplia distribución intercontinental (Figura 3). Desagregado por regiones, la mayoría de las 171 especies presentes en la región mediterránea de la península proceden de la Cuenca Mediterránea (25\%), Eurasia (19\%) y Europa (14\%). En la conformación de la mayoría (51\%) de las plantas no nativas distribuidas en el desierto se mantiene la proporción de las Eurasiáticas (19\%), disminuye significativamente el peso de la Cuenca del Mediterráneo (10\%) e incrementan el peso relativo África (11\%) y Norteamérica (10\%). En la región tropical, la procedencia de la región euroasiática mantiene un $14 \%$, se reduce drásticamente la presencia de especies de Europa (3\%) y de la Cuenca del Mediterráneo (3\%), y se incrementa significativamente la proporción de especies de África (19\%), Norteamérica (14\%), Sudamérica (12\%) y de especies procedentes de África y Asia (12\%) (Figura 4).

Formas de vida. Las especies herbáceas $(91 \%)$ son la forma de vida dominante entre las plantas exóticas naturalizadas, frente a tan sólo un 6\% de especies arbóreas y un 3\% de arbustivas. El patrón de dominancia se mantiene si se considera la distribución de las formas de vida dentro de cada una de las tres regiones de la península, pero con un incremento latitudinal en el peso de las especies leñosas (árboles y arbustos): mediterráneo $(4 \%)$, desierto $(15 \%)$ y región tropical (25\%) (Figura 5).

Ciclo de vida. Las plantas de ciclo de vida anual fueron las más numerosas (54\%), frente a un 34\% de perennes. El restante $12 \%$ de plantas exóticas posee ciclos de vida con distintas combinaciones de anualidad/bianualidad/perennidad. La distribución dentro de las tres regiones peninsulares fue semejante a la observada para la península en su conjunto,

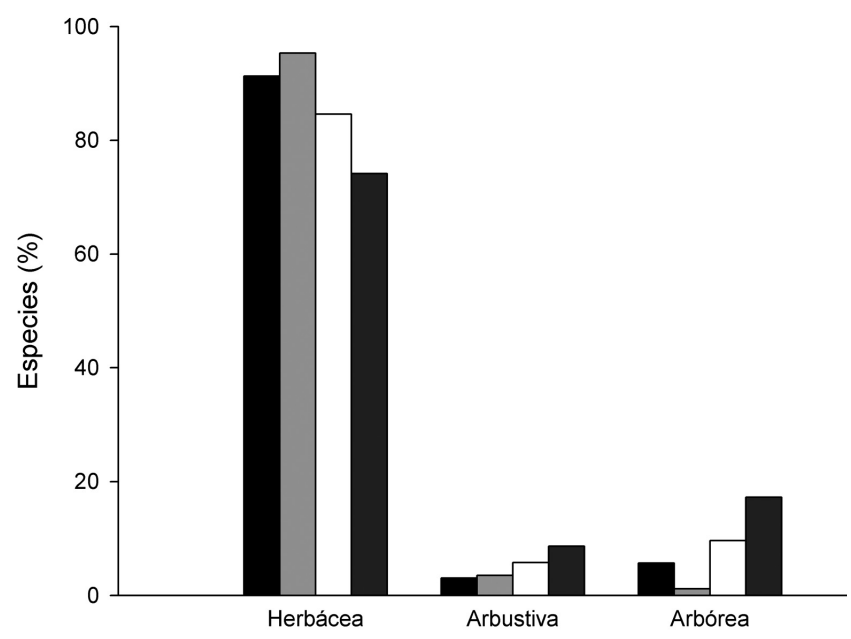

Figura 5. Distribución de las formas de vida de las plantas exóticas naturalizadas en el conjunto de la península (negro) y en cada una de las tres regiones peninsulares: mediterránea (gris claro), desértica (blanco) y tropical (gris oscuro). con un ligero incremento de las plantas perennes en la región tropical (43\%).

\section{Discusión}

Las 230 especies de flora exótica naturalizadas representarían el 7\% de la flora peninsular total, suponiendo que esta pudiera consistir al menos en unas 3,500 especies (J. Rebman, com. pers.). Esta proporción es inferior al $17.5 \%$ de flora exótica naturalizada encontrada en California (Qian et al., 2008), 14\% en la flora de Chile (Arroyo et al., 2000) o el $15 \%$ en la flora europea (Lambdon et al., 2008). Sin embargo, es similar al 6.7\% reportado para el estado de Sonora por Van Devender et al. (2010), al encontrado en diversos países de la Cuenca del Mediterráneo, como el 6.9\% en Italia (Celesti-Grapow et al., 2009), el 6.4\% en España (Lambdon et al., 2008); e incluso superior a lo observado en las partes más áridas del Mediterráneo: 2\% en Grecia (Arianoutsou et al., 2010) y entre 1.8-4.9\% en el norte de África (Vilà et al., 1999).

Aunque comparaciones generales de regiones tan variadas es aventurado, se considera en primer lugar, que la aridez existente en gran parte de la península es probablemente un fuerte condicionante para el establecimiento de especies exóticas, no sólo como limitante ecológico para las especies en sí (Vilà et al., 1999), sino como limitante para la intensidad de uso y ocupación humana del espacio, especialmente el agrícola. Por otro lado, la baja densidad poblacional, el relativo aislamiento humano de la península con el resto del continente, así como la escasez de vías de comunicación intrapeninsular hasta bien entrado el siglo XX (p. ej., la carretera transpeninsular se completó en 1973), han ralentizado probablemente la tasa de llegada de nuevas especies. La mayor presencia de especies exóticas en la región mediterránea peninsular, de condiciones menos áridas, mejor comunicada históricamente con el "exterior", especialmente el sur de California, y de reciente expansión agrícola, concuerda con esta línea argumental. La región mediterránea de California, a la que pertenece florísticamente el noroeste peninsular, ha sido reconocida como una región de alto grado de invasión de plantas (Fox, 1990; Rejmánek et al., 1991). El intenso crecimiento poblacional en el corredor costero del noroeste mediterráneo peninsular en las últimas décadas (300\% de incremento en la población urbana entre 1970-2010; INEGI, 2010, 2011), estrechamente conectado con el corredor urbano costero del otro lado de la frontera internacional, ha incrementado probablemente el transporte de especies desde el sur de California. Durante el mismo periodo se ha producido también en el resto de la península, aunque no en la misma intensidad, un cambio significativo en la geografía de la ocupación humana del territorio. Hubo un incremento de la población (INEGI, 2010), mejora de las vías de comunicación internas y la conexión aérea y marítima con el exterior (p. ej., la región tropical de Los Cabos 
con California y México). Este cambio de la geografía humana probablemente ha reducido la barrera biogeográfica que representaba el aislamiento peninsular y ha permitido la llegada de nuevas especies. Finalmente, no hay que olvidar el posible efecto que puede tener el menor conocimiento de la flora exótica peninsular, comparativamente con las regiones citadas, muy especialmente de las floras de ambientes urbanizados del norte y noroeste peninsular (Mexicali, Tecate, corredor costero Tijuana-Ensenada-San Quintín) y del extremo sur (La Paz, corredor turístico de Los Cabos).

La proporción de las especies exóticas que llegan a una nueva región y se naturalizan ha sido un elemento de gran interés y discusión (Williamson y Fitter, 1996). Las estimaciones reportadas sugieren que es muy variable y puede oscilar, por ejemplo, desde el 41-68\% de la flora exótica total en diversos países mediterráneos (Arianoutsou et al., 2010), hasta el $80 \%$ en México (Villaseñor y García-Espinosa, 2004). De estos valores podríamos inferir que el número total de plantas exóticas naturalizadas presentes actualmente en la península podría ser un $25-40 \%$ superior a las 230 especies reportadas en este trabajo (295-425 especies).

La flora exótica establecida en la península de Baja California está dominada por cuatro familias (57\%): Poaceae $(31 \%)$, Asteraceae (13\%), Brassicaceae (8\%) y Chenopodiaceae $(5 \%)$. Esta distribución taxonómica concuerda con el patrón encontrado en múltiples regiones del mundo (Pyšek, 1998), en diversas regiones de Norteamérica (Villaseñor y Espinosa-García, 2004; Van Devender et al., 2009; Hrusa et al., 2002; Dean et al., 20089), Sudamérica (Arroyo et al., 2000; Ugarte et al., 2011), Europa (Pyšek et al., 2002; Lambdon et al., 2008; Celesti-Grapow et al., 2009) o Asia (Weber et al., 2008; Wu et al., 2010; Khuroo et al., 2012). La abundancia de especies exóticas pertenecientes a las familias Asteraceae y Poaceae está posiblemente relacionada con el hecho de que son dos de las familias más numerosas (Pyšek, 1998). Es destacable la preponderancia de la familia Poaceae que contiene casi un tercio de la totalidad de especies naturalizadas en la península de Baja California. Estas especies se han introducido en calidad de cultivos, ornamentales o forrajeros, y muchas de ellas escapan de los cultivos y llegan a naturalizarse (Rzedowski y Calderón de Rzedowski 1990). Esta familia es también la más numerosa en lugares semiáridos y áridos como Grecia (Arianoutsou et al., 2010), Norte de África (Vilà et al., 1999), Chile (Arroyo et al., 2000), Sonora (Van Devender et al., 2009) y los desiertos de California (Kemp y Brooks, 1998). Sin embargo, la familia Asteraceae aparece como dominante en floras exóticas de regiones como Europa (Lambdon et al., 2008), India (Khuroo et al., 2012) o China (Wu et al., 2010).

La mayoría de las especies no nativas presentes en la península proceden del Viejo Mundo, especialmente de la Cuenca del Mediterráneo y Eurasia. La importancia de estas regiones de origen ha sido observado en las floras exóticas tanto de Norteamérica (Villaseñor y Espinosa-García, 2004; Van
Devender et al., 2009), como de Sudamérica (Arroyo et al., 2000). La explicación parece deberse en gran medida a la historia de expansión de la población europea después del siglo XV. En Europa, especialmente en la Cuenca Mediterránea, la prolongada exposición a las actividades humanas estimuló las adaptaciones al disturbio humano por parte de plantas nativas. Posteriormente, la secuencia histórica de la expansión humana, a partir del siglo XV, convirtió a Europa y a la Cuenca del Mediterráneo en las regiones exportadoras de colonizadores a distintas partes del Globo, pero también de sus ecosistemas asociados (Crosby 1986; La Sorte et al., 2007).

Sin embargo, es interesante señalar que junto a este patrón de origen de la flora exótica peninsular en su conjunto, parece existir cierto filtro ejercido por la región peninsular receptora (Figura 4). La proporción de especies procedentes de Europa y de la Cuenca del Mediterráneo muestran un peso relativo claramente diferenciado, y en orden decreciente, según se trate de la región mediterránea, el desierto o el área tropical. En cambio, aquellas procedentes de África, Norteamérica y Sudamérica presentan un patrón inverso de importancia relativa, creciente en la secuencia región mediterránea, desierto y área tropical. Esto sugeriría que aunque las relaciones históricas de intercambio humano entre regiones son el factor quizá de mayor peso sobre las especies intercambiadas, las condiciones ambientales de las regiones de destino también ejercen cierto grado de control sobre el establecimiento de nuevas especies.

Las herbáceas son la forma de vida dominante en la península, patrón que suele encontrarse en otras floras exóticas (Pyšek et al., 2002; Weber et al., 2008; Lambdon et al., 2008; Van Devender et al., 2009; Arianoutsou et al., 2010). La aridez de la península constituye sin duda una fuerte limitante para el establecimiento de formas de vida arbóreas y arbustivas (Vilà et al., 1999). En la región tropical del sur peninsular, donde las condiciones de aridez se atenúan comparativamente, se incrementa ligeramente la proporción de arbustos y árboles exóticos. El escaso número de especies exóticas arbóreas o arbustivas en la región mediterránea coincide con el patrón de invasión observado en las cinco regiones mediterráneas a nivel mundial (Fox, 1990), con una primera fase temporal en la que el intercambio de especies exóticas estaría dominado por el movimiento de especies herbáceas desde la Cuenca del Mediterráneo hacia las otras cuatro regiones mediterráneas.

El 8\% de la flora naturalizada en la península de Baja California tiene comportamiento invasor. Esta proporción es semejante al 5-6\% de plantas invasoras estimadas en la flora exótica de California (Bossard et al., 2000; Dark, 2004), pero muy inferior al 15\% de la flora no nativa de Sonora (Van Devender et al., 2009). Dado que la categorización aquí presentada no se basa en un procedimiento sistematizado, como en el caso de California (Cal-IPC, 2006, 2007), la lista de especies invasoras de este trabajo puede tener un sesgo sub- 
jetivo y distorsionar su comparación con otras estimaciones. Sería deseable, en lo posible, seguir protocolos sistematizados para identificar especies invasoras, como en el caso de California (Cal-IPC, 2006, 2007) o Arizona (Northam et al., 2005), que permitan disponer listas comparables de estas especies. La región mediterránea peninsular contiene mayor proporción de plantas invasoras (16 del total de 18 especies) que el desierto y el trópico, reúnen nueve y seis especies, respectivamente. Además de las plantas observadas como invasoras en la península, existe un grupo de especies naturalizadas que han sido categorizadas como invasoras en las regiones circundantes de California (Cal-IPC, 2006, 2007), Arizona (Northam et al., 2005) y Sonora (Van Devender et al., 2009) (ver Apéndice). Estas especies son en principio candidatas a comportamiento invasor en la península, por lo que deberían ser consideradas, al menos precautoriamente, en las acciones de conservación y manejo. Finalmente, ocho de las especies incluidas en el Apéndice (Carduus pycnocephalus, Ehrharta erecta, Erodium botrys, Gastrydium phleoides, Limonium perezii, Lythrum hyssopifolia, Malephora crocea y Myoporum laetum) son nuevas citas para México, y no están registradas en el inventario de plantas exóticas publicado para el conjunto de México por Villaseñor y Espinosa-García (2004). El esfuerzo realizado para construir el primer listado de plantas exóticas a nivel de todo el país merece que se acompañe con trabajos regionales y locales que ayuden a complementar y actualizar el conocimiento de la flora exótica de México. Su naturaleza dinámica, temporal y espacial, requiere de la suma de múltiples esfuerzos a distintios niveles. En este contexto, el presente trabajo no pretende ser una lista cerrada, sino un punto de partida sobre el que seguir sumando esfuerzos de actualización de la flora exótica de la península, y que al mismo tiempo incentive estudios sobre las implicaciones ecológicas, evolutivas o biogeográficas de la flora foránea en la península de Baja California y en el conjunto de México.

\section{Agradecimientos}

Agradecemos a Charlotte González-Abraham su ayuda en la elaboración de las figuras. Este trabajo fue parcialmente financiado por The Nature Conservancy. José Luis Villaseñor y un revisor anónimo realizaron comentarios que ayudaron a mejorar notoriamente el presente trabajo.

\section{Literatura citada}

Arianoutsou M., Bazos I., Delipetrou P. y Kokkoris Y. 2010. The alien flora of Greece: taxonomy, life traits and habitat preferences. Biological Invasions 12:3525-3549.

Arroyo M.T.K., Marticorena C., Matthei O. y Cavieres L. 2000. Plant invasions in Chile: present patterns and future predictions. En: Mooney H.A. y Hobbs H.A. Eds. Invasive Species in a Changing World, pp. 385-421, Island Press, Washington D.C.

Aschmann H. 1959. The Central Desert of Baja California: De- mography and Ecology. The University of California Press, Riverside.

Baker H.G. y Stebbins G.L. 1965. The Genetics of Colonizing Species. Academic Press Inc., Nueva York.

Bossard, C.C., Randall, J.M. y Hoshovsky M.C. 2000. Invasive Plants of California's Wildlands. University of California Press, Los Angeles.

Cal-IPC. 2006. California Invasive Plant Inventory. Cal-IPC Publication 2006-2012. California Invasive Plant Council. Berkeley. < http://www.cal-ipc.org/ip/inventory/pdf/Inventory2006. pdf $>$ (consultado enero 2010).

Cal-IPC. 2007. Inventory Update. California Invasive Plant Council. Berkeley. < http://www.cal-ipc.org/ip/inventory/pdf/WebUpdate2007.pdf> (consultado enero 2010)

Celesti-Grapow L., Alessandrini A., Arrigoni P.V., Banfi E., Bernardo L., Bovio M., Brundu G., Cagiotti M.R., Camarda I., Carli E., Conti F., Fascetti S., Galasso G., Gubellini L., La Valva V., Lucchese F., Marchiori S., Mazzola P., Peccenini S., Poldini L., Pretto F., Prosser F., Siniscalco C., Villani M.C., Viegi L., Wilhalm T. y Blasi C. 2009. Inventory of the non-native flora of Italy. Plant Biosystems 143:386-430.

Crosby A.W. 1986. Ecological Imperialism: The Biological Expansion of Europe, 900-1900. Cambridge University Press, Cambridge.

Dark S.J. 2004. The biogeography of invasive alien plants in California: an application of GIS and spatial regression analysis. Diversity and Distributions 10:1-9.

Davis L.G. 2003. Geoarchaeology and geochronology of pluvial Lake Chapala, Baja California, Mexico. Geoarchaeology 18:205-223.

Davis M.A., Grime J.P. y Thompson K. 2000. Fluctuating resources in plant communities: a general theory of invasibility. Journal of Ecology 88:528-534.

Davis M.A., Thompson K. y Grime J.P. 2005. Invasibility: the local mechanism driving community assembly and species diversity. Ecography 28:696-704.

Dean E., Hrusa F., Leppig G., Sanders A. y Ertter B. 2008. Catalogue of nonnative vascular plants occurring spontaneously in California beyond those addressed in the Jepson manual - Part II. Madroño 55:93-112.

Deasy G.F. y Gerhard P. 1944. Settlements in Baja California: 1768-1930. Geographical Review 34: 574-586.

del Barco, M. C. 1780. Correcciones y adiciones a la historia o noticia de la California en su primera edición de Madrid, año de 1757. (Editado por León-Portilla M. como Historia Natural y Crónica de la Antigua California, Instituto de Investigaciones Históricas, Universidad Nacional Autónoma de México, 1988, México D.F.).

eFloras 2011. Missouri Botanical Garden, St. Louis, MO \& Harvard University Herbaria, Cambridge, MA <www.efloras.org> (consultado junio-diciembre 2011).

Ehrenfeld J.G. 2010. Ecosystem consequences of biological invasions. Annual Review of Ecology, Evolution, and Systematics 41:59-80.

Elton C.S. 1958. The Ecology of Invasions by Animals and Plants. Methuen, Londres.

Espinosa García F.J. 2000. Malezas Introducidas en México. Centro de Investigaciones en Ecosistemas, Universidad Autónoma de México. Bases de datos SNIB-CONABIO proyecto U024. México, D.F. 
Espinosa-García F.J., Villaseñor J.L. y Vibrans H. 2004a. The rich generally get richer, but there are exceptions: Correlations between species richness of native plant species and alien weeds in Mexico. Diversity and Distributions 10:399-407.

Espinosa-García F.J., Villaseñor J.L. y Vibrans H. 2004b. Geographical patterns in native and exotic weeds of Mexico. Weed Technology 18:1552-1558.

Fox M.D. 1990. Mediterranean weeds: exchanges of invasive plants between the five Mediterranean regions of the world. En: di Castri F., Hansen A.J. y Debussche M. Eds. Biological Invasions in Europe and the Mediterranean Basin, pp. 179-200, Kluwer Academic Publishers, Dordrecht.

Fujita H. 2006. The Cape region. En: Laylander D. y Moore J.D. Eds. The Prehistory of Baja California: Advances in the Archaeology of the Forgotten Peninsula, pp. 82-98, University Press of Florida, Gainesville.

Garcillán P.P., González-Abrahamm C.E. y Ezcurra E. 2010. The cartographers of life: Two centuries of mapping the natural history of Baja California. Journal of the Southwest 52:1-40.

González-Abraham C.E., Garcillán P.P., Ezcurra E. y El Grupo de Trabajo de Ecorregiones. 2010. Ecorregiones de la península de Baja California: Una síntesis. Boletín de la Sociedad Botánica de México 87:69-82.

Gould F.W. y Moran R. 1981. The Grasses of Baja California, Mexico. San Diego Society of Natural History, San Diego.

Groves R.H., Panetta F.D. y Virtue J.G. 2001. Weed Risk Assessment. CSIRO Publishing, Collingwood.

Hastings J.R. y Turner R.M. 1965. Seasonal precipitation regimes in Baja California, Mexico. Geografiska Annaler. Series A 47:204-223.

Hrusa F., Ertter B., Sanders A., Leppig G. y Dean E. 2002. Catalogue of non-native vascular plants occurring spontaneously in California beyond those addressed in The Jepson manual-Part 1. Madroño 49:61-98.

Hulme P.E., Roy D.B., Cunha T. y Larsson T. 2009. A pan-European inventory of alien species: rationale, implementation and implications for managing biological invasions. En: DAISIE Ed. Handbook of Alien Species in Europe, pp 1-13, Springer, Berlín.

INEGI. 2010. Estadísticas Históricas de México 2009. Instituto Nacional de Estadística y Geografía, Aguascalientes.

INEGI. 2011. Anuario de Estadísticas por Entidad Federativa 2011. Instituto Nacional de Estadística y Geografía, Aguascalientes.

Kemp P. y Brooks M.L. 1998. Exotic species of California deserts. Fremontia 26:30-34.

Khuroo A.A., Reshi Z., Rashid I., Dar G.H. y Khan Z.S. 2008. Operational characterization of alien invasive flora and its management implications. Biodiversity and Conservation 17:3181-3194.

Khuroo A.A., Reshi Z.A., Malik A.H., Weber E., Rashid I. y Dar G.H. 2012. Alien flora of India: taxonomic composition, invasion status and biogeographic affiliations. Biological Invasions 14:99-113.

Lambdon P.W., Pyšek P., Basnou C., Hejda M., Arianoutsou M., Essl F., Jarošík V., Pergl J., Winter M., Anastasiu P., Andriopoulos P., Bazos I., Brundu G., Celesti-Grapow L., Chassot P., Delipetrou P., Josefsson M., Kark S., Klotz S., Kokkoris Y., Kühn I., Marchante H., Perglová I., Pino J., Vilà M., Zikos A., Roy D. y Hulme P.E. 2008. Alien flora of Europe: species diversity, temporal trends, geographical patterns and research needs. Preslia 80:101-149.
La Sorte F.A., McKinney M.L. y Pyšek P. 2007. Compositional similarity among urban floras within and across continents: biogeographical consequences of human-mediated biotic interchange. Global Change Biology 13:913-921.

León de la Luz J.L., Domínguez-León M., y Van Devender T. 2009. Baja California Sur: Native, exotic and invasive weeds. En: Van Devender T.R., Espinosa-García F.J., Harper-Lore B.L. y Hubbard T. Eds. Invasive Plants on the Move. Controlling them in North America Based on Presentations from Weeds Across Borders 2006 Conference, pp. 125-136, Arizona-Sonora Desert Museum, Tucson.

Mack R.N., Simberloff D., Lonsdale W.M., Evans H., Clout M. y Bazzaz F.A. 2000. Biotic invasions: causes, epidemiology, global consequences, and control. Ecological Applications 10:689710 .

Mack R.N. y Lonsdale W.M. 2001. Humans as global plant dispersers: Getting more than we bargained for. BioScience 51:95102

McMahon S.M., Cadotte M.W. y Fukami T. 2006. Trackling the tractable: Using invasions to guide the exploration of conceptual ecology. En: Cadotte M.W., McMahon S.M. y Fukami T. Eds. Conceptual Ecology and Invasion Biology: Reciprocal Approaches to Nature, pp. 3-14, Springer, Dordrecht.

Meyerson L.A. y Mooney H.A. 2007. Invasive alien species in an era of globalization. Frontiers in Ecology and the Environment 5:199-208.

Mooney H.A. y Hobbs R.J. Eds. 2000. Invasive Species in a Changing World. Island Press, Washington, DC.

Northam F.E., Backer D.M. y Hall J.A. 2005. Development of a categorized list of invasive non-native plants that threaten wildlands in Arizona. Final report of the Arizona Wildlands Invasive Plant Working Group. <http://sbsc.wr.usgs.gov/research/ projects/swepic/SWVMA/DevelopmentOfACategorizedList. pdf $>$ (consultado 9 septiembre 2007).

Pimentel D., Zuniga R. y Morrison D. 2005. Update on the environmental and economic costs associated with alien-invasive species in the United States. Ecological Economics 52:273288.

Pimentel D., Lach L., Zuniga R. y Morrison D. 2000. Environmental and economic costs of nonindigenous species in the United States. BioScience 50:53-65.

Pyšek P. 1998. Is there a taxonomic pattern to plant invasions? Oikos 82:282-294.

Pyšek P. y Richardson D.M. 2006. The biogeography of naturalization in alien plants. Journal of Biogeography 33:2040-2050.

Pyšek P. y Richardson D.M. 2007. Traits associated with invasiveness in alien plants: where do we stand? En: Nentwig W. Ed. Biological Invasions, Ecological Studies 193, pp. 97-125, Springer-Verlag, Berlín y Heidelberg.

Pyšek P., Sádlo J., y Mandák B. 2002. Catalogue of alien plants of the Czech Republic. Preslia Praha 74:97-186.

Pyšek P., Sádlo J., Mandák B. y Jarošik V. 2003. Czech alien flora and the historical pattern of its formation: what came first to Central Europe? Oecologia 135:122-130.

Pyšek P., Richardson D.M., Rejmánek M., Webster G.L., Williamson M. y Kirschner J. 2004. Alien plants in checklists and floras: towards better communication between taxonomists and ecologists. Taxon 53:131-143.

Pyšek P., Jarošik V., Hulme P.E., Kühn I., Wild J., Arianoutsou M., Bacher S., Chiron F., Didžiulis V., Essl F., Genovesi P., Ghe- 
rardi F., Hejda M., Kark S., Lambdon P.W., Desprez-Loustau M.L., Nentwig W., Pergl J., Poboljšaj K., Rabitsch W., Roques A., Roy D.B., Shirley S., Solarz W., Vilà M. y Winter M. 2010. Disentangling the role of environmental and human pressures on biological invasions across Europe. Proceedings of the $\mathrm{Na}$ tional Academy of Sciences USA 107:12157-12162.

Qian H., McKinney M.L. y Kühn I. 2008. Effects of introduced species on floristic similarity: Comparing two US states. Basic and Applied Ecology 9:617-625.

Rejmánek M., Thomsen C.D. y Peters I.D. 1991. Invasive vascular plants of California. En: Groves R.H. y Di Castri F. Eds. Biogeography of Mediterranean Invasions, 81-102, Cambridge University Press, Cambridge.

Richardson D.M., Pyšek P., Rejmanek M., Barbour M.G., Panetta F.D. y West C.J. 2000. Naturalization and invasion of alien plants: Concepts and definitions. Diversity and Distributions 6:93-107.

Richardson D.M. y Pyšek P. 2006. Plant invasions: merging the concepts of species invasiveness and community invasibility. Progress in Physical Geography 30:409-431.

Richardson D.M. Ed. 2011. Fifty Years of Invasion Ecology: The Legacy of Charles Elton. Wiley-Blackwell, Oxford.

Rzedowski J. y Calderón de Rzedowski G. 1990. Nota sobre el elemento africano en la flora adventicia de México. Acta Botanica Mexicana 12:21-24.

Sax D.F. 2001. Latitudinal gradients and geographic ranges of exotic species: implications for biogeography. Journal of Biogeography 28:139-150.

Sax D.F. y Gaines S.D. 2003. Species diversity: from global decreases to local increases. Trends in Ecology and Evolution 18:561-566.

Sax D.F., Stachowicz J.J. y Gaines S.D. Eds. 2005. Species Invasions. Insights into Ecology, Evolution, and Biogeography. Sinauer Associates, Sunderland.

Sol D., Vilà M. y Kühn I. 2008. The comparative analysis of historical alien introductions. Biological Invasions 10:1119-1129.

Sutherland S. 2004. What makes a weed a weed: life history traits of native and exotic plants in the USA. Oecologia 141:24-39.

Taylor B.W. y Irwin R.E. 2004. Linking economic activities to the distribution of exotic plants. Proceedings of the National Academy of Sciences USA 101:17725-17730.

Turner II B.L., Clark W.C., Kates R.W., Richards J.F., Mathews J.T. y Meyer W.B. 1990. The Earth as Transformed by Human
Action: Global and Regional Changes in the Biosphere over the Past 300 Years. Cambridge University Press, Cambridge.

Ugarte E., Lira F., Fuentes N. y Klotz S. 2011. Vascular alien flora, Chile. Check List 7:365-382.

Van Devender T.R., Felger R.S., Reina-Guerrero A.L. y SánchezEscalante J.J. 2009. Sonora: Non-native and invasive plants. En: Van Devender T.R., Espinosa-García F.J., Harper-Lore B.L. y Hubbard T. Eds. Invasive Plants on the Move. Controlling them in North America. Based on Presentations from Weeds Across Borders 2006 Conference, pp. 85-124, Arizona-Sonora Desert Museum, Tucson.

Van Devender T.R., Felger R.S., Fishbein M., Molina-Freaner F.E., Sánchez-Escalante J.J. y Reina-Guerrero A.L. 2010. Biodiversidad de las plantas vasculares. En: Molina-Freaner F.E. y Van Devender T.R. Eds. Diversidad Biológica de Sonora, pp. 229261, Universidad Nacional Autónoma de México, México D.F.

Vibrans H. 2011. Malezas de México. En línea: <www.conabio. gob.mx/malezasdemexico/2inicio/home-malezas-mexico.htm> (consultado junio-diciembre 2011).

Vilà M., Meggaro Y. y Weber E. 1999. Preliminary analysis of the naturalized flora of northern Africa. Orsis 14:9-20.

Vilà M., Basnou C., Pyšek P., Josefsson M., Genovesi P., Gollasch S., Nentwig W., Olenin S., Roques A., Roy D., Hulme P.E. y DAISIE partners. 2009. How well do we understand the impacts of alien species on ecosystem services? A pan-European cross-taxa assessment. Frontiers in Ecology and the Environment 8:135-144.

Villaseñor J.L. y Espinosa-García F.J. 2004. The alien flowering plants of Mexico. Diversity and Distributions 10:113-123.

Vitousek P.M., Mooney H.A., Lubchenco J. y Melillo J.M. 1997. Human domination of earth's ecosystems. Science 277:494499.

Weber E., Sun S.G. y Li B. 2008. Invasive alien plants in China: diversity and ecological highlights. Biological Invasions 8:1411-1429.

Wiggins I.L. 1980. Flora of Baja California. Stanford University Press, Palo Alto.

Williamson M. y Fitter A. 1996. The varying success of invaders. Ecology 77:1661-1666.

Wu S.H., Sun H.T., Teng Y.C., Rejmánek M., Chaw S.M., Yang T.Y.A. y Hsieh C.F. 2010. Patterns of plant invasions in China: taxonomic, biogeographic, climatic approaches and anthropogenic effects. Biological Invasions 12:2179-2206.

Recibido: 6 de noviembre de 2012.

Aceptado: 20 de febrero de 2013. 
Apéndice. Relación de plantas no nativas naturalizadas de la península de Baja California (PBC). Se indica su origen biogeográfico (Af: África, As: Asia, AuAs: Australasia, CM: Cuenca del Mediterráneo, Cri: Criptogénicas, Eu: Europa, EuAs: Eurasia, NAf: Norte de África, NAm: Norteamérica, SAf: África del Sur, SAm: Sudamérica). En la columna "Nat. en Mx" se indican aquellos taxones nativos de México cuya distribución natural sugeriría que son también nativos en la PBC, pero que son considerados como exóticos en este trabajo. Se muestran las regiones peninsulares donde están preferentemente distribuidas cada una de las plantas (M: Mediterránea, D: Desértica, T: Tropical). Plantas observadas en la península de Baja California con carácter invasor (I) y categorización de estatus invasor reportado (H: alto, M: medio, L: bajo) en regiones circundantes de Arizona (Ari; Northam et al., 2005) y California (Cal; Cal-IPC, 2006, 2007), y Sonora (Son; I: invasora; Van Devender et al., 2009).

\begin{tabular}{|c|c|c|c|c|c|c|c|c|}
\hline \multirow[t]{2}{*}{ Taxón } & \multirow[t]{2}{*}{ Familia } & \multirow[t]{2}{*}{ Origen } & \multirow{2}{*}{$\begin{array}{c}\text { Nat. } \\
\text { en } \\
\text { Mx }\end{array}$} & \multirow{2}{*}{$\begin{array}{l}\text { Dist. } \\
\text { PBC }\end{array}$} & \multicolumn{4}{|c|}{ Estatus invasor } \\
\hline & & & & & PBC & Ari. & Cal. & Son. \\
\hline
\end{tabular}

Acacia cochliacantha Humb. \& Bonpl. ex Wild.

Acacia farnesiana (L.) Willd.

Agrostis stolonifera L.

Aloe vera (L.) Burm. f.

Amaranthus albus L.

Amaranthus cruentus L.

Amaranthus deflexus L.

Amaranthus hybridus L.

Amaranthus retroflexus $\mathrm{L}$.

Amaranthus spinosus L.

Amaranthus viridis L.

Ambrosia artemisiifolia L.

Anagallis arvensis L.

Anthemis cotula L.

Apium graveolens L.

Arundo donax L.

Asclepias curassavica L.

Atriplex lindleyi Moq.

Atriplex rosea L.

Atriplex semibaccata R.Br.

Atriplex suberecta I. Verd.

Avena barbata Pott ex Link

Avena fatua L.

Avena sativa L.

Bassia hyssopifolia (Pall.) Kuntze

Beta vulgaris L.

Bidens pilosa L.

Brachypodium distachyon (L.) P.Beauv.

Brassica juncea (L.) Czern.

Brassica nigra (L.) W.D.J.Koch

Brassica rapa L. subsp. sylvestris (L.) Janch. \& Wendelb.

Brassica tournefortii Gouan

Bromus berteroanus Colla

Bromus briziformis Fisch. \& C.A.Mey.

Bromus catharticus Vahl

Bromus diandrus Roth

Bromus hordeaceus L.

Bromus madritensis L. subsp. madritensis

\begin{tabular}{|c|c|}
\hline Fabaceae & NAm \\
\hline Fabaceae & NAm-SAm \\
\hline Poaceae & EuAs \\
\hline Asphodelaceae & NAf \\
\hline Amaranthaceae & $\mathrm{NAm}$ \\
\hline Amaranthaceae & SAm \\
\hline Amaranthaceae & SAm \\
\hline Amaranthaceae & NAm \\
\hline Amaranthaceae & $\mathrm{NAm}$ \\
\hline Amaranthaceae & NAm-SAm \\
\hline Amaranthaceae & SAm \\
\hline Asteraceae & NAm \\
\hline Myrsinaceae & EuAs \\
\hline Asteraceae & Eu-NAf \\
\hline Apiaceae & EuAs \\
\hline Poaceae & EuAs \\
\hline Apocynaceae & NAm-SAm \\
\hline Chenopodiaceae & $\mathrm{Au}$ \\
\hline Chenopodiaceae & Af-EuAs \\
\hline Chenopodiaceae & $\mathrm{Au}$ \\
\hline Chenopodiaceae & $\mathrm{Au}$ \\
\hline Poaceae & $\mathrm{CM}$ \\
\hline Poaceae & EuAs \\
\hline Poaceae & $\mathrm{Eu}$ \\
\hline Chenopodiaceae & EuAs \\
\hline Chenopodiaceae & Cri \\
\hline Asteraceae & Nam-SAm \\
\hline Poaceae & $\mathrm{Eu}$ \\
\hline Brassicaceae & EuAs \\
\hline Brassicaceae & $\mathrm{Eu}$ \\
\hline Brassicaceae & EuAs \\
\hline Brassicaceae & $\mathrm{CM}$ \\
\hline Poaceae & NAm-SAm \\
\hline Poaceae & EuAs \\
\hline Poaceae & SAm \\
\hline Poaceae & $\mathrm{CM}$ \\
\hline Poaceae & EuAs \\
\hline Poaceae & $\mathrm{CM}$ \\
\hline
\end{tabular}

$\mathrm{X} \quad \mathrm{T}$

$X \quad M$

$M$

MDT

$\mathrm{T}$

$\mathrm{T}$

D

T

$\mathrm{D}$

$\mathrm{T}$

$\mathrm{T}$

X D

$\mathrm{T}$

M

$M$

$\begin{array}{lllll}\text { MDT I } & \mathrm{H} & \mathrm{H} & \text { I }\end{array}$

M

$M$

MD

$M$

$M$

$M$

$M$

$M$

$M$

M

$M$

$\mathrm{MD}$

MD

$\mathrm{MD}$

$\mathrm{MD}$

M

$M$

$M$

$M$

$M$

M 
Apéndice. Continuación

\begin{tabular}{|c|c|c|c|c|c|c|c|c|}
\hline \multirow[t]{2}{*}{ Taxón } & \multirow[t]{2}{*}{ Familia } & \multirow[t]{2}{*}{ Origen } & \multirow{2}{*}{$\begin{array}{c}\text { Nat. } \\
\text { en } \\
\text { Mx }\end{array}$} & \multirow{2}{*}{$\begin{array}{l}\text { Dist. } \\
\text { PBC }\end{array}$} & \multicolumn{4}{|c|}{ Estatus invasor } \\
\hline & & & & & PBC & Ari. & Cal. & Son. \\
\hline Bromus madritensis L. subsp. rubens (L.)Husn. & Poaceae & $\mathrm{CM}$ & & M & I & $\mathrm{H}$ & $\mathrm{H}$ & I \\
\hline Bromus tectorum $\mathrm{L}$. & Poaceae & EuAs & & M & & $\mathrm{H}$ & $\mathrm{H}$ & \\
\hline Caesalpinia gilliesii (Wall. ex Hook.) D. Dietr. & Fabaceae & SAm & & M & & & & \\
\hline Cakile maritima Scop. & Brassicaceae & $\mathrm{Eu}$ & & MD & & & $\mathrm{L}$ & \\
\hline Capsella bursa-pastoris (L.) Medik. & Brassicaceae & EuAs & & MD & & & & \\
\hline Carduus pycnocephalus L. & Asteraceae & $\mathrm{CM}$ & & M & & & & \\
\hline Carpobrotus chilensis (Molina) N.E.Br. & Aizoaceae & SAf & & M & I & & M & \\
\hline Carpobrotus edulis (L.) N.E.Br. & Aizoaceae & SAf & & M & I & & $\mathrm{H}$ & \\
\hline Cenchrus echinatus L. & Poaceae & NAm-SAm & $X$ & $\mathrm{D}$ & & & & \\
\hline Cenchrus incertus M.A.Curtis & Poaceae & NAm & $\mathrm{X}$ & $\mathrm{D}$ & & & & \\
\hline Centaurea benedicta (L.) L. & Asteraceae & $\mathrm{Eu}$ & & M & & & & \\
\hline Centaurea cyanus L. & Asteraceae & $\mathrm{Eu}$ & & M & & & & \\
\hline Centaurea melitensis L. & Asteraceae & $\mathrm{CM}$ & & M & I & M & M & \\
\hline Centaurea solstitialis L. & Asteraceae & $\mathrm{CM}$ & & MD & & $\mathrm{H}$ & $\mathrm{H}$ & \\
\hline Cerastium glomeratum Thuill. & Caryophyllaceae & $\mathrm{Eu}$ & & M & & & & \\
\hline Chenopodium album L. & Chenopodiaceae & EuAs-NAf & & MD & & & & \\
\hline Chenopodium glaucum L. & Chenopodiaceae & Cri & & M & & & & \\
\hline Chenopodium macrospermum Hook. $\mathrm{f}$. & Chenopodiaceae & SAm & & M & & & & \\
\hline Chenopodium murale L. & Chenopodiaceae & $\mathrm{Eu}$ & & MDT & & & & \\
\hline Chloris barbata Sw. & Poaceae & Cri & & $\mathrm{D}$ & & & & \\
\hline Chloris gayana Kunth & Poaceae & Af & & DT & & & & \\
\hline Cirsium vulgare (Savi) Ten. & Asteraceae & $\mathrm{Eu}$ & & MD & & $\mathrm{L}$ & M & \\
\hline Cleome viscosa $\mathrm{L}$. & Cleomaceae & As & & DT & & & & 1 \\
\hline Convolvulus arvensis $\mathrm{L}$. & Convolvulaceae & $\mathrm{Eu}$ & & MD & & M & & 1 \\
\hline Coriandrum sativum $\mathrm{L}$. & Apiaceae & $\mathrm{CM}$ & & M & & & & \\
\hline Cotula australis (Sieber ex Spreng.) Hook. f. & Asteraceae & $\mathrm{Au}$ & & M & & & & \\
\hline Cotula coronopifolia L. & Asteraceae & SAf & & M & & & $\mathrm{L}$ & \\
\hline Crypsis schoenoides (L.) Lam. & Poaceae & $\mathrm{Eu}$ & & M & & & & \\
\hline Crypsis vaginiflora (Forssk.) Opiz & Poaceae & EuAs & & $M$ & & & & \\
\hline Cryptostegia grandiflora (Roxb.) R.Br. & Apocynaceae & Af & & MDT & I & & & I \\
\hline Cucumis dipsaceus Ehrenb. & Cucurbitaceae & Af & & MDT & & & & \\
\hline $\begin{array}{l}\text { Cyclospermum leptophyllum (Pers.) Sprague } \\
\text { ex Britton \& P.Wilson }\end{array}$ & Apiaceae & Nam-SAm & $X$ & $\mathrm{D}$ & & & & \\
\hline Cynara cardunculus L. & Asteraceae & $\mathrm{CM}$ & & $M$ & & & & \\
\hline Cynodon dactylon (L.) Pers. & Poaceae & Af & & MDT & & M & M & 1 \\
\hline Cyperus cuspidatus Kunth & Cyperaceae & $\begin{array}{l}\text { Af-As-Au- } \\
\text { NAm-SAm }\end{array}$ & & $M$ & & & & \\
\hline Cyperus involucratus Rottb. & Cyperaceae & Af & & M & & & & \\
\hline Cyperus rotundus $\mathrm{L}$. & Cyperaceae & EuAs & & $\mathrm{D}$ & & & & \\
\hline Dactyloctenium aegyptium (L.) Willd. & Poaceae & Af-As & & MDT & & & & \\
\hline Descurainia sophia (L.) Webb ex Prantl & Brassicaceae & EuAs & & MD & & & $\mathrm{L}$ & 1 \\
\hline Digitaria bicornis (Lam.) Roem. \& Schult. & Poaceae & As & & DT & & & & \\
\hline Digitaria ciliaris (Retz.) Koeler & Poaceae & Cri & & DT & & & & \\
\hline Digitaria sanguinalis (L.) Scop. & Poaceae & EuAs & & DT & & & & \\
\hline
\end{tabular}


Apéndice. Continuación

\begin{tabular}{|c|c|c|c|c|c|c|c|c|}
\hline \multirow[t]{2}{*}{ Taxón } & \multirow[t]{2}{*}{ Familia } & \multirow[t]{2}{*}{ Origen } & \multirow{2}{*}{$\begin{array}{c}\text { Nat. } \\
\text { en } \\
\text { Mx }\end{array}$} & \multirow{2}{*}{$\begin{array}{l}\text { Dist. } \\
\text { PBC }\end{array}$} & \multicolumn{4}{|c|}{ Estatus invasor } \\
\hline & & & & & PBC & Ari. & Cal. & Son. \\
\hline Diplotaxis muralis (L.) DC. & Brassicaceae & $\mathrm{Eu}$ & & M & & & & \\
\hline Dysphania ambrosioides (L.) Mosyakin \& Clemants & Chenopodiaceae & NAm-SAm & $X$ & M & & & & \\
\hline Echinochloa colona (L.) Link & Poaceae & EuAs & & DT & & & & \\
\hline Echinochloa crus-galli (L.) P.Beauv. & Poaceae & Cri & & MD & & $\mathrm{L}$ & & \\
\hline Ehrharta erecta Lam. & Poaceae & SAf & & $M$ & & & M & \\
\hline Eleusine indica (L.) Gaertn. & Poaceae & Af-As & & MDT & & & & \\
\hline Elymus ponticus (Podp.) N.Snow & Poaceae & EuAs & & $M$ & & & & \\
\hline Eragrostis cilianensis (All.) Vignolo ex Janch. & Poaceae & $\mathrm{CM}$ & & MDT & & & & \\
\hline Eragrostis ciliaris (L.) R.Br. & Poaceae & Af-As & & MDT & & & & \\
\hline Eragrostis minor Host & Poaceae & EuAs & & $\mathrm{D}$ & & & & \\
\hline Eragrostis tenella (L.) P.Veauv. ex Roem. \& Schult. & Poaceae & Af-As & & MT & & & & \\
\hline Eragrostis viscosa (Retz.) Trin. & Poaceae & Af-As & & $\mathrm{D}$ & & & & \\
\hline Erigeron bonariensis L. & Asteraceae & SAm & & MD & & & & \\
\hline Erodium botrys (Cav.) Bertol. & Geraniaceae & $\mathrm{CM}$ & & $M$ & & & & \\
\hline Erodium brachycarpum (Godr.) Thell. & Geraniaceae & $\mathrm{Eu}$ & & $M$ & & & & \\
\hline Erodium cicutarium (L.) L'Hér. ex Aiton & Geraniaceae & EuAs & & M & & M & $\mathrm{L}$ & \\
\hline Erodium moschatum (L.) L'Hér. ex Aiton & Geraniaceae & EuAs & & M & & & & \\
\hline Eruca vesicaria (L.) Cav. subsp. sativa (Mill.) Thell. & Brassicaceae & EuAs & & MD & & & & I \\
\hline Euphorbia maculata L. & Euphorbiaceae & NAm & & $\mathrm{D}$ & & & & \\
\hline Euphorbia serpens Kunth & Euphorbiaceae & NAm-SAm & $x$ & $\mathrm{D}$ & & & & \\
\hline Festuca bromoides L. & Poaceae & Af-Eu & & M & & & & \\
\hline Festuca myuros L. & Poaceae & EuAs & & M & & & M & \\
\hline Festuca ovina L. & Poaceae & EuAs & & $M$ & & & & \\
\hline Festuca perennis (L.) Columbus \& J.P.Sm. & Poaceae & $\mathrm{CM}$ & & MD & & M & M & \\
\hline Festuca temulenta (L.) Columbus \& J.P.Sm. & Poaceae & $\mathrm{Eu}$ & & MD & & & & \\
\hline Foeniculum vulgare Mill. & Apiaceae & Eu-NAf & & M & I & & $\mathrm{H}$ & \\
\hline Gastridium phleoides (Nees \& Meyen) C.E.Hubb. & Poaceae & $\mathrm{CM}$ & & M & & & & \\
\hline Glebionis coronaria (L.) Spach & Asteraceae & $\mathrm{CM}$ & & M & I & & M & \\
\hline Hackelochloa granularis (L.) Kuntze & Poaceae & Af-As-Au & & $\mathrm{D}$ & & & & \\
\hline Hainardia cylindrica (Willd.) Greuter & Poaceae & Af-EuAs & & M & & & & \\
\hline Hedypnois cretica (L.) Dum. Cours. & Asteraceae & $\mathrm{CM}$ & & M & & & & \\
\hline $\begin{array}{l}\text { Herniaria hirsuta L. subsp. cinerea (DC.) } \\
\text { Loret \& Barrandon }\end{array}$ & Caryophyllaceae & Af-EuAs & & M & & & & \\
\hline Heteropogon melanocarpus (Elliott) Benth. & Poaceae & As & & DT & & & & \\
\hline Hirschfeldia incana (L.) Lagr.-Fossat & Brassicaceae & $\mathrm{CM}$ & & M & & & M & \\
\hline $\begin{array}{l}\text { Hordeum marinum Huds. subsp. gussoneanum } \\
\text { (Parl.) Thell }\end{array}$ & Poaceae & Af-EuAs & & $M$ & & & & \\
\hline Hordeum murinum L. subsp. glaucum (Steud.) Tzvelev & Poaceae & $\mathrm{CM}$ & & M & & M & M & \\
\hline Hordeum murinum L. subsp. leporinum (Link) Arcang. & Poaceae & $\mathrm{CM}$ & & M & & M & M & \\
\hline Hypochaeris glabra L. & Asteraceae & Eu-NAf & & $M$ & & & $\mathrm{~L}$ & \\
\hline Lactuca serriola L. & Asteraceae & $\mathrm{Eu}$ & & MD & & & & \\
\hline Lamarckia aurea (L.) Moench & Poaceae & $\mathrm{CM}$ & & M & & & & \\
\hline Lavatera arborea L. & Malvaceae & $\mathrm{CM}$ & & MD & & & & \\
\hline Leonotis nepetifolia (L.) R.Br. & Lamiaceae & Af & & MDT & & & & \\
\hline
\end{tabular}


Apéndice. Continuación

\begin{tabular}{|c|c|c|c|c|c|c|c|c|}
\hline \multirow[t]{2}{*}{ Taxón } & \multirow[t]{2}{*}{ Familia } & \multirow[t]{2}{*}{ Origen } & \multirow{2}{*}{$\begin{array}{c}\text { Nat. } \\
\text { en } \\
\text { Mx }\end{array}$} & \multirow{2}{*}{$\begin{array}{l}\text { Dist. } \\
\text { PBC }\end{array}$} & \multicolumn{4}{|c|}{ Estatus invasor } \\
\hline & & & & & PBC & Ari. & Cal. & Son. \\
\hline Leucaena leucocephala (Lam.) de Witt & Fabaceae & NAm & $X$ & DT & & & & I \\
\hline Limonium perezii (Stapf) F.T.Hubb. & Plumbaginaceae & Af & & M & & & & \\
\hline Limonium sinuatum (L.) Mill. & Plumbaginaceae & $\mathrm{CM}$ & & M & & & & \\
\hline Lobularia maritima (L.) Desv. & Brassicaceae & $\mathrm{CM}$ & & $M$ & & & $\mathrm{~L}$ & \\
\hline Logfia gallica (L.) Coss. \& Germ. & Asteraceae & $\mathrm{CM}$ & & $M$ & & & & \\
\hline Lythrum hyssopifolia L. & Lythraceae & EuAs & & M & & & $\mathrm{L}$ & \\
\hline Malephora crocea (Jacq.) Schwantes & Aizoaceae & SAf & & M & & & & \\
\hline Malva assurgentiflora (Kellogg) M.F. Ray & Malvaceae & NAm & $X$ & MD & & & & \\
\hline Malva parviflora L. & Malvaceae & $\mathrm{CM}$ & & $M$ & & & & \\
\hline Malva sylvestris L. & Malvaceae & EuAs-NAf & & M & & & & \\
\hline Marrubium vulgare L. & Lamiaceae & EuAs-NAf & & M & & & $\mathrm{L}$ & \\
\hline Matricaria discoidea DC. & Asteraceae & Cri & & M & & & & \\
\hline Matthiola incana (L.) W.T.Aiton & Brassicaceae & $\mathrm{CM}$ & & M & & & & \\
\hline Medicago polymorpha L. & Fabaceae & EuAs-NAf & & M & & & $\mathrm{L}$ & \\
\hline Melilotus albus Medik. & Fabaceae & EuAs & & $M$ & & M & & \\
\hline Melilotus indicus (L.) All. & Fabaceae & EuAs & & $M$ & & & & \\
\hline Melinis repens (Willd.) Zizka subsp. repens & Poaceae & Af & & $\mathrm{T}$ & & & & I \\
\hline Mentha aquatica L. & Lamiaceae & Eu-NAf & & $M$ & & & & \\
\hline Mentha arvensis L. & Lamiaceae & EuAs & & $M$ & & & & \\
\hline Mentha spicata L. & Lamiaceae & $\mathrm{CM}$ & & M & & & & \\
\hline Mesembryanthemum crystallinum L. & Aizoaceae & SAf & & MD & & $\mathrm{L}$ & M & $\mathrm{I}$ \\
\hline Mesembryanthemum nodiflorum L. & Aizoaceae & SAf & & MD & I & M & & \\
\hline Momordica charantia L. & Cucurbitaceae & Af-As & & MD & & & & \\
\hline Myoporum laetum G.Forst. & Scrophulariaceae & $\mathrm{Au}$ & & $M$ & I & & & \\
\hline Nicandra physalodes (L.) Gaertn. & Solanaceae & Nam-SAm & $X$ & M & & & & \\
\hline Nicotiana glauca Graham & Solanaceae & SAm & & MDT & & & M & I \\
\hline Oenothera curtiflora W.L.Wagner \& Hoch & Onagraceae & NAm & & $\mathrm{D}$ & & & & \\
\hline Oxalis pes-caprae L. & Oxalidaceae & SAf & & M & & & M & \\
\hline Panicum antidotale Retz. & Poaceae & As & & $\mathrm{T}$ & & $\mathrm{L}$ & & \\
\hline Panicum purpurascens Raddi & Poaceae & Af & & MDT & & & & \\
\hline Parapholis incurva (L.) C.E.Hubb. & Poaceae & Af-EuAs & & $M$ & & & & \\
\hline Parkinsonia aculeata L. & Fabaceae & Nam-SAm & $X$ & DT & & & & 1 \\
\hline Paspalum dilatatum Poir. & Poaceae & SAm & & $M$ & & & & \\
\hline Paspalum urvillei Steud. & Poaceae & SAm & & M & & & & \\
\hline Pelargonium X hortorum L.H.Bailey & Geraniaceae & SAf & & $M$ & & & & \\
\hline Pennisetum ciliare (L.) Link & Poaceae & Af & & MDT & I & $\mathrm{H}$ & & 1 \\
\hline Pennisetum setaceum (Forssk.) Chiov. & Poaceae & Af & & $M$ & & $\mathrm{H}$ & M & $\mathrm{I}$ \\
\hline Persicaria maculosa Gray & Polygonaceae & EuAs & & $\mathrm{T}$ & & & & \\
\hline Phalaris caroliniana Walter & Poaceae & NAm & & $M$ & & & & \\
\hline Phalaris minor Retz. & Poaceae & $\mathrm{CM}$ & & $M$ & & & & \\
\hline Phalaris paradoxa L. & Poaceae & $\mathrm{CM}$ & & M & & & & \\
\hline Phoenix dactylifera L. & Arecaceae & As & & DT & & & & \\
\hline Physalis nicandroides Schltdl. & Solanaceae & NAm & $x$ & DT & & & & \\
\hline Phytolacca icosandra L. & Phytolaccaceae & NAm & $X$ & $M$ & & & & \\
\hline
\end{tabular}


Apéndice. Continuación

\begin{tabular}{|c|c|c|c|c|c|c|c|c|}
\hline \multirow[t]{2}{*}{ Taxón } & \multirow[t]{2}{*}{ Familia } & \multirow[t]{2}{*}{ Origen } & \multirow{2}{*}{$\begin{array}{l}\text { Nat. } \\
\text { en } \\
\text { Mx }\end{array}$} & \multirow{2}{*}{$\begin{array}{l}\text { Dist. } \\
\text { PBC }\end{array}$} & \multicolumn{4}{|c|}{ Estatus invasor } \\
\hline & & & & & PBC & Ari. & Cal. & Son. \\
\hline Picris echioides L. & Asteraceae & $\mathrm{CM}$ & & $\mathrm{D}$ & & & $\mathrm{L}$ & \\
\hline Pithecellobium dulce (Roxb.) Benth. & Fabaceae & NAm & $X$ & DT & & & & \\
\hline Plantago lanceolata L. & Plantaginaceae & EuAs & & MD & & & $\mathrm{L}$ & \\
\hline Plantago major L. & Plantaginaceae & EuAs & & MD & & & & \\
\hline Poa annua L. & Poaceae & $\mathrm{Eu}$ & & M & & & & \\
\hline Poa pratensis L. & Poaceae & $\mathrm{CM}$ & & M & & & $\mathrm{L}$ & \\
\hline Polycarpon tetraphyllum (L.) L. & Caryophyllaceae & $\mathrm{CM}$ & & M & & & & \\
\hline Polygonum argyrocoleon Steud. ex Kunze & Polygonaceae & EuAs & & MD & & & & \\
\hline $\begin{array}{l}\text { Polygonum aviculare L. subsp. depressum } \\
\text { (Meisn.) Arcang. }\end{array}$ & Polygonaceae & $\mathrm{Eu}$ & & M & & & & \\
\hline $\begin{array}{l}\text { Polygonum aviculare L. subsp. neglectum } \\
\text { (Besser) Arcang. }\end{array}$ & Polygonaceae & $\mathrm{Eu}$ & & MDT & & & & \\
\hline Polypogon australis Brongn. & Poaceae & SAm & & M & & & & \\
\hline Polypogon interruptus Kunth & Poaceae & SAm & & MD & & & & \\
\hline Polypogon monspeliensis (L.) Desf. & Poaceae & Af-EuAs & & MD & & & $\mathrm{L}$ & I \\
\hline Polypogon viridis (Gouan) Breistr. & Poaceae & $\mathrm{CM}$ & & M & & & & \\
\hline Portulaca oleracea L. & Portulacaceae & Cri & & DT & & & & \\
\hline $\begin{array}{l}\text { Pseudognaphalium luteoalbum (L.) Hilliard } \\
\quad \& \text { B.L.Burtt }\end{array}$ & Asteraceae & EuAs & & $\mathrm{MD}$ & & & & \\
\hline Raphanus raphanistrum L. & Brassicaceae & $\mathrm{CM}$ & & M & & & & \\
\hline Raphanus sativus L. & Brassicaceae & $\mathrm{CM}$ & & M & & & $\mathrm{L}$ & \\
\hline Rhaponticum repens (L.) Hidalgo & Asteraceae & EuAs & & M & & $\mathrm{H}$ & M & \\
\hline Ricinus communis L. & Euphorbiaceae & Af & & MDT & I & & $\mathrm{L}$ & I \\
\hline Robinia pseudoacacia L. & Fabaceae & NAm & & M & & & & \\
\hline Rumex conglomeratus Murray & Polygonaceae & Af-EuAs & & $\mathrm{D}$ & & & & \\
\hline Rumex crispus L. & Polygonaceae & EuAs & & $\mathrm{D}$ & & & $\mathrm{L}$ & \\
\hline Rumex obtusifolius L. & Polygonaceae & Af-EuAs & & MD & & & & \\
\hline Rumex pulcher L. & Polygonaceae & $\mathrm{CM}$ & & MD & & & & \\
\hline Rumex acetosella L. & Polygonaceae & EuAs & & MD & & & & \\
\hline Ruta chalepensis L. & Rutaceae & $\mathrm{CM}$ & & M & & & & \\
\hline Salsola tragus $\mathrm{L}$. & Chenopodiaceae & $\mathrm{Eu}$ & & MD & I & M & $\mathrm{L}$ & I \\
\hline Schinus molle L. & Anacardiaceae & SAm & & DT & & & $\mathrm{L}$ & \\
\hline Schinus terebinthifolius Raddi & Anacardiaceae & SAm & & DT & I & & $\mathrm{L}$ & \\
\hline Schismus barbatus (L.) Thell. & Poaceae & Af-EuAs & & $\mathrm{D}$ & & M & $\mathrm{L}$ & I \\
\hline Senecio vulgaris $\mathrm{L}$. & Asteraceae & $\mathrm{Eu}$ & & M & & & & \\
\hline Sesbania herbacea (Mill.) McVaugh & Fabaceae & NAm & $x$ & DT & & & & \\
\hline Setaria adhaerens (Forssk.) Chiov. & Poaceae & $\begin{array}{l}\text { Af-EuAs- } \\
\text { NAm-SAm }\end{array}$ & & DT & & & & \\
\hline Setaria verticillata (L.)P.Beauv. & Poaceae & $\mathrm{Eu}$ & & DT & & & & \\
\hline Setaria viridis (L.) Beauv. & Poaceae & EuAs & & DT & & & & \\
\hline Silene gallica L. & Caryophyllaceae & $\mathrm{Eu}$ & & M & & & & \\
\hline Silybum marianum (L.) Gaertn. & Asteraceae & $\mathrm{CM}$ & & M & & & $\mathrm{L}$ & \\
\hline Sisymbrium altissimum L. & Brassicaceae & As & & MD & & & & \\
\hline Sisymbrium irio L. & Brassicaceae & $\mathrm{CM}$ & & MD & & & $M$ & \\
\hline
\end{tabular}


Apéndice. Continuación

\begin{tabular}{|c|c|c|c|c|c|c|c|c|}
\hline \multirow[t]{2}{*}{ Taxón } & \multirow[t]{2}{*}{ Familia } & \multirow[t]{2}{*}{ Origen } & \multirow{2}{*}{$\begin{array}{c}\text { Nat. } \\
\text { en } \\
\text { Mx }\end{array}$} & \multirow{2}{*}{$\begin{array}{l}\text { Dist. } \\
\text { PBC }\end{array}$} & \multicolumn{4}{|c|}{ Estatus invasor } \\
\hline & & & & & PBC & Ari. & Cal. & Son. \\
\hline Sisymbrium officinale (L.) Scop. & Brassicaceae & EuAs & & $M$ & & & & \\
\hline Sisymbrium orientale L. & Brassicaceae & $\mathrm{Eu}$ & & $M$ & & & & \\
\hline Solanum elaeagnifolium Cav. & Solanaceae & NAm-SAm & $X$ & $M$ & & & & \\
\hline Solanum nigrum $\mathrm{L}$. & Solanaceae & EuAs & & MT & & & & \\
\hline Sonchus asper (L.) Hill & Asteraceae & EuAs & & MD & & M & & \\
\hline Sonchus oleraceus L. & Asteraceae & $\mathrm{Eu}$ & & MD & & M & & \\
\hline Sonchus tenerrimus L. & Asteraceae & $\mathrm{CM}$ & & MD & & & & \\
\hline Sorghum bicolor (L.) Moench & Poaceae & Af & & DT & & & & \\
\hline Sorghum halepense (L.) Pers. & Poaceae & Af-As & & DT & & M & & I \\
\hline Spergularia bocconi (Scheele) Graebn. & Caryophyllaceae & $\mathrm{CM}$ & & M & & & & \\
\hline Spergularia villosa (Pers.) Cambess. & Caryophyllaceae & SAm & & M & & & & \\
\hline Stellaria media (L.) Vill. & Caryophyllaceae & EuAs & & MD & & & & \\
\hline Stenotaphrum secundatum (Walter) Kuntze & Poaceae & Af-As-SAm & & M & & & & \\
\hline Tamarix aphylla (L.) Karsten & Tamaricaceae & Af-As & & DT & & $\mathrm{L}$ & $\mathrm{L}$ & I \\
\hline Tamarix chinensis Lour. & Tamaricaceae & As & & $\mathrm{D}$ & & $\mathrm{H}$ & & I \\
\hline Tamarix parviflora DC. & Tamaricaceae & $\mathrm{CM}$ & & DT & & $\mathrm{H}$ & $\mathrm{H}$ & \\
\hline Tamarix ramosissima Ledeb. & Tamaricaceae & EuAs & & DT & I & $\mathrm{H}$ & $\mathrm{H}$ & \\
\hline Tanacetum parthenium (L.) Sch.Bip. & Asteraceae & $\mathrm{Eu}$ & & M & & & & \\
\hline Taraxacum officinale Wigg. & Asteraceae & EuAs & & M & & & & \\
\hline Tetragonia tetragonioides (Pall.) Kuntze & Aizoaceae & As-Au & & M & & & & \\
\hline Tragus berteronianus Schult. & Poaceae & Af-As & & MDT & & & & \\
\hline Tribulus terrestris $\mathrm{L}$. & Zygophyllaceae & Af-EuAs & & DT & & & & \\
\hline Tropaeolum majus L. & Tropaeolaceae & SAm & & M & & & & \\
\hline Urtica urens L. & Urticaceae & $\mathrm{Eu}$ & & $M$ & & & & \\
\hline $\begin{array}{l}\text { Verbesina encelioides (Cav.) Benth. \& Hook. f. } \\
\text { ex A.Gray subsp. encelioides }\end{array}$ & Asteraceae & NAm & $X$ & DT & & & & \\
\hline Waltheria indica $\mathrm{L}$. & Malvaceae & NAm-SAm & $X$ & DT & & & & \\
\hline Xanthium spinosum L. & Asteraceae & SAm & & DT & & & & \\
\hline
\end{tabular}

